\title{
Group behaviour in physical, chemical and biological systems
}

\author{
Cihan Saçlioğlu ${ }^{1}$, Önder Pekcan ${ }^{2}$ and Vidyanand Nanjundiah ${ }^{3}$ \\ ${ }^{I}$ Mühendislik ve Doğa Bilimleri Fakültesi, Sabancı Üniversitesi, Tuzla, İstanbul 34956, \\ Turkey \\ ${ }^{2}$ Mühendislik ve Doğa Bilimleri Fakültesi, Kadir Has Üniversitesi, Cibali, İstanbul 34083, \\ Turkey \\ ${ }^{3}$ Centre for Ecological Sciences, Indian Institute of Science, Bangalore 560 012, India
}

(Emails,CS-saclioglu@sabanciuniv.edu; OP-pekcan@khas.edu.tr; VN-vidya@ces.iisc.ernet.in)

\begin{abstract}
Groups exhibit properties that either are not perceived to exist, or perhaps cannot exist, at the individual level. Such 'emergent' properties depend on how individuals interact, both among themselves and with their surroundings. The world of everyday objects consists of material entities. These are, ultimately, groups of elementary particles that organize themselves into atoms and molecules, occupy space, and so on. It turns out that an explanation of even the most commonplace features of this world requires relativistic quantum field theory and the fact that Planck's constant is discrete, not zero. Groups of molecules in solution, in particular polymers ('sols'), can form viscous clusters that behave like elastic solids ('gels'). Sol-gel transitions are examples of cooperative phenomena. Their occurrence is explained by modelling the statistics of inter-unit interactions: the likelihood of either state varies sharply as a critical parameter crosses a threshold value. Group behaviour among cells or organisms is often heritable and therefore can evolve. This permits an additional, typically biological, explanation for it in terms of reproductive advantage, whether of the individual or of the group. There is no general agreement on the appropriate explanatory framework for understanding group-level phenomena in biology.
\end{abstract}

[Saçlığlu C, Pekcan Ö and Nanjundiah V 2014 Group behaviour in physical, chemical and biological systems. J. Biosci. 39 177-189] DOI $10.1007 / \mathrm{s} 12038-013-9398-4$

\section{Introduction}

For understanding group-level phenomena in the living world, a pragmatic course would be to begin by asking, in respect of a particular manifestation, whether it can be understood on the basis of principles that have been used successfully to explain group behaviour in the non-living world. Only when that fails should one look for a specifically biological explanation. ${ }^{1}$ In this article we illustrate the contrasting ways in which the behaviour of groups is viewed in the natural sciences. The few examples that are chosen are by no means comprehensive. Their purpose is to illustrate

\footnotetext{
${ }^{1}$ 'Rule out geometric, then physical and then chemical explanations before invoking biologically specific ones' (The Life of a Leaf by Steven Vogel; University of Chicago Press, 303 pp, 2012).
}

how the study of groups is approached in physics, chemistry and biology, in particular the different levels at which explanations are sought. ${ }^{2}$

Natural science began in the attempt to understand phenomena. 'Understand' encompasses observing, the ordering of impressions, experimentation, hypothesis-building, comparing rival hypotheses and so on - what is called the 'scientific method'. ${ }^{3}$ The phenomena that attracted most

\footnotetext{
${ }^{2}$ This sequence of dealing with the three areas is traditional and reflects the often-quoted assertion that 'all chemistry is physics and all biology is chemistry'.

${ }^{3}$ That we are able to understand the world at all, that by and large things happen as we expect them to, is because we - meaning all living creatures - are products of evolution. See http://www.iep.utm.edu/evoepis/ for a short introduction to evolutionary epistemology.
}

Keywords. Adaptation; cooperation; epidemic; gelation; identical particles; Pauli Exclusion Principle; percolation; relativistic quantum field theory; representations of the Poincare group; social selection; stability and identity of DNA; swelling 
attention from this viewpoint occurred at spatial scales and over times that were suited to human perception. In the course of time it was realized that what was perceived could be described in terms of the behaviour of basic units whose size is too small, and whose states change too rapidly, to be perceived directly. Exactly what the units are is a pragmatic matter. Atoms (physics), molecules (chemistry) and cells (biology) are typical examples. But in each field, the unit can be smaller or larger - it all depends on the problem to be addressed. For instance, physicists also deal with elementary particles and planets as basic units, chemists with atoms and crystals, and biologists with molecules and multicellular organisms.

Sometimes it happens that the behaviour of a large system can be thought of as a trivial extension of the summed behaviour of its constituent units; in such cases the saying 'the whole is the sum of its parts' applies. However, simple summation no longer works if nonlinear effects are significant, as can happen when there are strong interactions between the parts. Then the whole gives the impression of being 'more than the sum of its parts'; the whole has to be considered as an entity on its own. ${ }^{4}$ It displays emergent properties, properties that are qualitatively different from those of its constituents. A molecule of sodium chloride is altogether different from an atom of sodium and an atom of chlorine. We will look at how physicists, biologist and chemists try to come to grips with properties that emerge as the number of constituent units in a system goes from one to many. ${ }^{5}$

Depending on the property of the system being monitored, one can expect the following consequences of size increase: ${ }^{6}$ (a) An intensive property, such as density, will show no change. (b) An extensive or colligative property will change in a manner proportional to the number or concentration; for example, the rise in boiling point of water brought about by dissolving a substance. (c) There may be a disproportionate change with number or size as in the allometric growth exhibited by many leaves. (d) An emergent property may become apparent as the number increases, as for example in a phase transition. A standard example of a phase transition in chemistry is the sol-to-gel transition. Here a substance that can polymerize, when dissolved in a second substance, forms an extended cluster (of 'infinite' size) beyond a threshold concentration of the solute. Below the threshold one has at most local aggregates of the solute. The infinitely large macromolecule is an amorphous solid,

\footnotetext{
${ }^{4}$ It can also be that the number of parts is so large that summing up their properties is no longer a feasible exercise (as with the molecules of gas in a room). Then one settles for a statistical description of the whole. What we mean here is something different: even in principle it is impossible to look at the whole as a number of non-interacting parts.

${ }^{5}$ Meaning number of constituent units.

${ }^{6}$ Again, meaning one brought about by an increase in the number of subunits or components.
}

a gel; the collection of finite clusters is a sol, a complex entity that retains properties of a fluid. ${ }^{7}$

Physical and chemical groups exhibit a range of behaviours, not all of them obvious from what we know of their components. In particular, they can exhibit stability (longterm persistence) and variation (alternative stable outcomes). Heterogeneity and inter-unit interactions make variation more likely and, possibly, transitions between states less likely. For groups at equilibrium the criteria that determine the outcome are intrinsic to the system (e.g. the state of lowest internal energy). ${ }^{8}$ The physico-chemical approach to studying the behaviour of biological groups is based on viewing biology as the science of living matter. In other words, biological systems are in the first instance material entities made up of definable physical units. The units display a natural hierarchy that can be expressed in the following sequence: small molecules $\rightarrow$ macromolecules $\rightarrow$ supramolecular aggregates $\rightarrow$ organelles $\rightarrow$ cells $\rightarrow$ tissues $\rightarrow$ individuals $\rightarrow$ species. ${ }^{9}$ Behaviour that is relevant to the whole is expressed at each of these levels. In certain respects it resembles the sorts of group behaviour in physics and chemistry that we have considered so far. Therefore, it is reasonable to expect it to be explainable on similar principles. But an aspect of behaviour that is peculiar to living matter demarcates biology from physics and chemistry: cells and individuals can reproduce. ${ }^{10}$ This property, along with that of variation, makes it possible for a mode of long-term evolutionary change that is unique to biology, known as natural selection. Natural selection endows living creatures with features that appear to be the result of design, of a longterm goal. Because of this, there is the possibility of understanding biological systems not just on the basis of how physical and chemical principles can account for their structures, but also on the basis of why particular structures have come about. We will refer to an answer to the 'why' question, whether or not it involves natural selection, ${ }^{11}$ as a biological explanation for the trait or phenomenon of

\footnotetext{
${ }^{7}$ The sol-gel transition is an example of a percolation transition and critical behaviour.

${ }^{8}$ It has been conjectured that systems close to equilibrium follow the rule of minimum rate of entropy production. We know of no thermodynamic principle that can act as a guide to predicting the state of a system far from equilibrium.

${ }^{9}$ There are also individuals consisting of one cell; in such situations 'tissue' lacks meaning.

${ }^{10}$ In the case of a cell 'reproduction' implies the production of a second cell ('daughter' or 'offspring') identical - in a sense to be discussed later to the first ('parent'). In a different form of reproduction, known as sexual reproduction, the offspring can be derived from two dissimilar individuals. In that case it resembles its parents but is distinct from both of them.

${ }^{11}$ Biological form and structure may also be explicable on a physical basis, at least with regard to its origins, and to that extent may not demand an explanation based on natural selection (Newman and Comper 1990)
} 
interest. A biological explanation is essentially a historical explanation. It hinges on something that originated in the (past) ancestry of the individual that exhibits the trait, and on the fact that the propensity to display the trait has been transmitted through descent. Our contention is not that historical explanations are unique to biology, but that they are central to biology more than to physics or chemistry. ${ }^{12}$

The rest of this article is organized as follows. The first section uses non-technical language to get across the point that if one wants to understand the material world, one is compelled to invoke the properties of its most fundamental constituents, the elementary particles. The phenomena displayed by macroscopic matter cannot be understood except as extensions of the properties of elementary particles. The implication is that quantum mechanics (in the form of relativistic quantum field theory) is central to understanding the world of direct experience. In particular this is true of the two most familiar properties of matter in the large, namely, that it is stable and occupies space. Collections of identical particles of integral or halfintegral spin display the simplest individual-to-group transitions exhibited by matter, and their behaviour too depends essentially on quantum mechanics. The widely held belief that classical physics remains valid in the limit in which Planck's constant (the smallest amount of angular momentum or action allowed by quantum mechanics) vanishes, is false.

The next section considers material entities at the larger level of molecules and points out that under certain conditions molecular groups exhibit phase transitions, namely, qualitative changes in properties similar to what happens when ice melts or water boils. A particular type of phase transition, the sol-gel transition, is used for illustration. Its most striking features are best explained at a phenomenological level, i.e. well above that of quantum fields or elementary particles; the link between phenomenology and quantum field theory is believed to be secure even if not always spelt out explicitly. The explanation rests on concepts such as interaction probabilities between atoms or molecules, the fact that the same macroscopic configuration can be consistent with many equivalent microscopic configurations and considerations of 'phase space' (Arpağ and Erzan 2014).

Already at this level it is possible to draw analogies between a material phenomenon and features of behaviour in biological groups. In fact, in an unusual case of biology informing physical science, methods used to study infectious disease epidemics can be usefully applied to throw light on the dynamics of the sol-gel phase transition. That brings us to biological groups and the question of what constitutes a specifically biological explanation for group behaviour. Here the explanatory framework partly relies on concepts that are

\footnotetext{
${ }^{12}$ The relative abundances of the chemical elements are explained by invoking a particular history of the universe; on a smaller time scale, the precise course of annealing can cause the same alloy to end up with drastically different properties.
}

easily visualized in terms of molecule-level properties (e.g. intercellular adhesion). Also, in part it invokes concepts at a yet higher level (e.g. the correlations between genes and behaviour) that - going down the scale - are believed to rest securely on organismal, cellular and molecular properties.

The large number of footnotes is aimed, first, at guiding the reader through widely disparate fields of study and, second, with pointing out exceptions or nuances that are glossed over in the main body of the text so as not to distract the reader. The article concludes with a brief summary.

\section{Physical principles underlying collective behaviour: Elementary particles and emergent macroscopic manifestations}

The basic facts of chemistry are often taken for granted and used to explain phenomena, with no attempt made to go into the quantum physics that underlies them. This works for two reasons: first of all, although relativistic quantum field theory (RQFT) is a more basic level of description of physical phenomena, many of the degrees of freedom (i.e. parameters required to specify the system, that can vary independently) of RQFT are 'frozen' at the energies relevant for biochemistry or biology, allowing a simpler description in terms of effective theories. Another reason is that given the ambient temperatures and the large number of atoms and molecules required in life processes, a phenomenon known as decoherence washes out the non-classical wavelike aspects of quantum phenomena. Nevertheless, in the 'classical' world there are still fundamental signatures of RQFT, many of which are responsible for the accepted facts of chemistry. It is worthwhile to identify and trace these down to their origins, thus filling the gap between the usual starting point for biological sciences and the currently accepted bottom of scientific reductionist hierarchy, built on elementary particles such as electrons, photons and quarks (which, bound together by the strong-force carriers, the gluons, make up protons and neutrons).

Experiments probing distances down to $10^{-18} \mathrm{~m}$ support the so-called 'Standard Model' of the weak, electromagnetic and strong interactions in which elementary particles participate (see 'The Standard Model' in The Physics Hypertextbook; http://physics.info/standard/). However, we will not try to argue that everything in Nature can be straightforwardly derived from this theory. The limitations of the strictly reductionist approach have been carefully described in Anderson's classic essay 'More is different' (Anderson 1972). Nevertheless, we will argue that familiar facts of the world of experience can be ultimately be traced back to RQFT (Saçlıoğlu 2003) combined with group theory. ${ }^{13}$

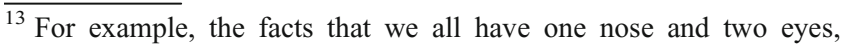
occupy a certain volume of space, and share many basic concepts number, common names and verbs.
} 
The choice of RQFT as a starting point merely reflects our current understanding of physics; it will almost certainly be demoted from its current 'fundamental' status if and when, say, a 'more fundamental' quantum theory that includes gravitation is found.

In his influential book What Is Life? Schrödinger (1944) argued that the stability, identity and discreteness of lifeforms originates from genes. In turn, genes derive these properties from the stability, identity and discreteness of molecules as dictated by quantum mechanics. Atoms are made of protons, neutrons and electrons. All chemistry results from the mediation of photons ${ }^{14}$ between atomic or molecular electrons. The identity, or at least classifiable similarity of everyday objects and phenomena, thus originates from this limited number of fundamental particles and their interactions. Identical, discrete building blocks lead to a set of identical or similar macroscopic objects such as apples, rocks and cats, rather than amorphous and unclassifiable matter. Thus, common names arise as a consequence of quantum mechanics (actually, of quantum field theory, as we shall see later) at the macroscopic level, and through such names, the concept of number naturally emerges as Frege (1884; Russell 1961) defined it: the number 3 is the property common to sets of three apples, three rocks, three cats and so on. These concepts, basic to the software of the human mind, are thus seen to be reflections of identical elementary particle properties. An alien, non-human intelligence looking at our world would presumably come up with similar terms or software to describe it. Our actual brain and nervous system, which constitute the hardware, are even more transparently dependent on atoms and chemistry in that they operate by the exchange of identical sodium, potassium and chloride ions across membranes - thus, roughly speaking, the identity of the ions ensures that brains and nervous systems work the same way. Now, the Schrödinger equation indeed guarantees that such ions will have the same properties if they are assembled from identical electrons and protons, but that raises the obvious question: why are all electrons in the universe the same?

The answer can be traced back to the empirical fact that that all points in space and all instants in time are equivalent (the former evidenced by momentum conservation and the latter by energy conservation), and also by the fact that there is no preferred inertial frame defining absolute rest, as established by the success of Special Relativity. ${ }^{15}$ The mathematical expression of these equivalences or symmetries is a

\footnotetext{
${ }^{14}$ Real or virtual photons. 'In physics, a virtual particle is a transient fluctuation that exhibits many of the characteristics of an ordinary particle, but that exists for a limited time' (http://en.wikipedia.org/ wiki/Virtual_particle).

${ }^{15}$ i.e. one cannot assign an absolute sense to the concept of rest or no movement; one can only say that a body is at rest (or is moving) relative to another.
}

set of elements and operations that constitute what is known as the Poincare group. Quantum mechanical wave functions of particles are concrete (i.e. usable) representations of the mathematical structure called the Poincare group (here 'group' is a mathematical term unrelated to the biological groups discussed in this issue). ${ }^{16}$ Wigner (1939) showed that the wave functions could be characterised by two labels designated $m^{2}$ and $s(s+1)$, where $m$ is the mass (raised to the second power because, strictly, what is meant is the square of the four-momentum, of which one part is the mass) and $s$ the intrinsic spin of the particle. The mathematics dictates that the parameter $m$ is continuous, while $s$ can only take the values $0,1 / 2,1,3 / 2,2,5 / 2$ and so on. A further step of quantization replaces the particles' wave functions by continuous functions of space and time known as quantum fields. ${ }^{17}$ Because the normal modes of these quantum field operators (which pervade all space and are 'eternal') can create and/or destroy quanta of the fields, i.e. the particles, an electron here and now has the same properties as one in a faraway galaxy billions of years ago. ${ }^{18}$ Wigner's classic essay 'The Unreasonable Effectiveness of Mathematics in the Natural Sciences' (Wigner 1960) contains further reflections on the mysterious fit between highly abstract mathematics and physical reality. ${ }^{19}$ The collective behaviour of identical particles and many basic features of everyday phenomena follow from combining Wigner's results with the 1948 spin-statistics theorem of Pauli (1940). This theorem, while technically difficult to prove and hard to explain in everyday terms, is based on very fundamental properties of nature, such as the positivity of energy, and the laws of physics being consistent with Einstein's relativity. So it applies universally to all particles, but has different consequences for integer spin and half-integer spin cases. It guarantees that integer spin particles such as photons must obey Bose-Einstein (BE) statistics, while those with half-integer spin are subject to Fermi-Dirac (FD) statistics. This means bosons tend to occupy the same quantum state and can 'act in concert', while fermions must obey the Pauli Exclusion Principle (PEP), meaning no two fermions can go into the same quantum state.

\footnotetext{
${ }^{16}$ In 'first quantized' quantum mechanics, position and momenta of particles become non-commuting operators. Their wave functions are representations of the Poincaré group, 'irreducible unitary' representations to be precise.

${ }^{17}$ Quantum field theory 'second quantized' quantum mechanics. The analogue of the classical field, e.g. the familiar electromagnetic field, is a set of non-commuting operators called quantum fields. The quantum field is a continuous function of space and time just as the classical one. 18 They are both created by the same electron quantum field that embodies Poincaré symmetry. We might say that all we see around us, including ourselves, are approximate and highly reducible product representations of the Poincaré group.

${ }^{19}$ A fit that is absent in biology (Nanjundiah 2005).
} 
To begin with bosons, the very recent report from CERN on the Higgs boson (CMS Collaboration 2012; Atlas Collaboration 2012), the last missing bit of the Standard Model, seems to clinch the view that the vacuum itself is a collective condensate - one may say the 'group' formed by spin-zero bosons, the 'individuals'. In addition, the most promising scheme for the confinement of quarks ${ }^{20}$ requires yet another universal condensate made of gluons. We inhabit these mediums and therefore are not directly aware of them. That apart, BE condensation is not a familiar everyday phenomenon. On earth, there are two gargantuan macroscopic examples, namely, the kilometer-long superfluid helium circulation system and the superconducting magnets at CERN, all kept at $1.9 \mathrm{~K}$. When liquid helium is cooled to $2.17 \mathrm{~K}$, it becomes a superfluid, i.e. flows with zero viscosity. This is because all the atoms have gone into the same lowest energy state and behave collectively. In superconductors, electron pairs are formed at a critical low temperature, creating effective spin-zero bosons. Subsequently, these bosons form a condensate which flows as an electrical current without resistance in response to an applied voltage. At the risk of drawing a misleading analogy, we point out that there are situations in biology in which interacting individuals constitute a group that displays qualitatively new behaviours, as, for instance, in the cellular slime moulds and social insects (Nanjundiah and Sathe 2011). A somewhat different everyday example of a BE condensate is coherent monochromatic light, which is perfectly realized in a laser beam. ${ }^{21}$ What classical electrodynamics textbooks call a monochromatic plane wave field is actually the collective wave function of these photons.

In contrast to the collective behaviour of bosons, identical fermions display a range of anti-collective (but highly coordinated) behaviours because of PEP. This is a ubiquitous feature of our everyday environment. In ascending order of scale, here are a few examples. Three quarks ${ }^{22}$ (fermions) form neutrons and protons. Free neutrons decay to protons, electrons and antineutrinos in about 10 minutes, but live practically forever in stable nuclei ${ }^{23}$ because the PEP allows no room for the new decay-product proton. This is a bit like becoming immortal because graveyards are already full! The atomic electrons bound to the nucleus have to fill subshells and shells. Atoms bond covalently to form molecules. The explanation of metallic electrical conductivity requires the PEP. At the macroscopic level, when water is poured into a glass, the level rises because of the PEP repulsion between the outer electrons of adjacent $\mathrm{H}_{2} \mathrm{O}$

\footnotetext{
${ }^{20}$ The mathematical demonstration of this is one of the million dollar Clay Institute problems.

${ }^{21}$ The photon chemical potential is zero at all temperatures. Therefore at any temperature such a beam can be thought of as a Bose-Einstein condensate of identical photons of indefinite number.

${ }^{22}$ With antisymmetric wavefunctions dictated by the Pauli Exclusion Principle (PEP).

${ }^{23}$ Because the Pauli Exclusion Principle forbids the resulting proton from going into one of the levels already occupied by other nuclear protons.
}

molecules. Thus, our 'classical' concepts of 'full' and 'empty' ultimately derive from RQFT, which in turn is based on Pauli's spin-statistics theorem and the kinematic symmetries of spacetime, mathematically expressed by the Poincaré group. Lieb and Thirring (1975; Lieb 1990) have given formal, rigorous proofs that the 'stability of matter from atoms to stars' can be understood in terms of a balance between attractive forces versus kinetic and PEP pressure. It is this balance that lets astronomical objects in certain mass ranges settle into stable final states like white dwarfs or neutron stars. Incidentally, the latter may contain superfluid cores, i.e. exhibit BE condensation effects (Pines and Alpar 1985). ${ }^{24}$

In all this, it is vital that Planck's constant $\hbar$, which has been defined earlier and is extremely tiny by macroscopic standards, is not exactly zero. Physically, it is clear that from all the preceding examples that the quantum-based properties of discreteness, stability and identity would be lost if $\hbar$ were truly zero. Such a universe (if imaginable at all) would be a collection of amorphous chunks of matter unprotected against gravitational collapse. This is clearly not the everyday world we often carelessly characterize as 'classical'. The limit also makes no sense mathematically: any quantum mechanical probability amplitude with classical action $S$ (roughly the time integral of the energy for the considered path of the particle) is proportional to the factor $\exp (i S / \hbar)$. The point $\hbar=0$ is an essential singularity of this function. No convergent small- $\hbar$ expansion around it is possible, as argued clearly by Berry (2002). Thus, while the results of classical physics can be recovered as approximations in the limit of very small $\hbar$, they cannot be recovered when $\hbar$ strictly equals zero. In the everyday macroscopic world with a small but non-vanishing $\hbar$ and $S \gg \hbar$, the quantum and classical descriptions are in agreement (except in the BE condensates, which are macroscopic but behave non-classically), but this definitely does not mean the classical world corresponds to $\hbar=0$ exactly. In short, quantum mechanics, and/or quantum field theory, cannot ever be 'turned off'. It is at the heart of all ordinary group-level phenomena seen in the physical world, not just what appear (to us) to be bizarre 'quantum phenomena'.

\section{Group behaviour in chemistry: Sols and gels}

In general, a phase transition is defined as the transformation of a thermodynamic system from one phase or state of matter to another. During a phase transition of a given medium, certain properties of the medium change, often discontinuously, as a result of some external condition, such as temperature, pressure, and others. According to the classical Ehrenfest criterion (Ehrenfest 1933), the order of a phase transition is determined by the lowest degree derivative of the relevant

\footnotetext{
${ }^{24}$ If the mass is above the Chandrasekhar limit, the star becomes a black hole.
} 
thermodynamic potential that is discontinuous at the transition. For example, for a change of state in a liquid at ambient pressure, when considering the Gibbs free energy as function of pressure and temperature, the first-order character of the transition is characterized, at the transition temperature, by a discontinuous variation of the entropy, which is related to the latent heat. The various solid/liquid/gas transitions are classified as first-order transitions because they involve a discontinuous change in density, which is the first derivative of the free energy with respect to chemical potential.

Second-order phase transitions are continuous in the first derivative (the order parameter, which is the first derivative of the free energy with respect to the external field, is continuous across the transition) but exhibit discontinuity in a second derivative of the free energy. These include the ferromagnetic phase transition in materials such as iron, where the magnetization, which is the first derivative of the free energy with respect to the applied magnetic field strength, increases continuously from zero as the temperature is lowered below the Curie temperature. The magnetic susceptibility, the second derivative of the free energy with the field, changes discontinuously. Superconducting and the superfluid transitions are also second-order phase transitions.

Since phase transitions are seen in diverse systems in nature, namely, in liquid crystals, in biological systems, in magnetic systems, in binary alloys, and in sol-gel transitions, they present a challenge in statistical mechanics and related areas (Stanley 1971; Ehrenfest 1933; Kulkarni et al. 2013 propose an interesting network-based comparison between the transformation of a normal cell to a cancerous cell and a phase transition).

Gels exemplify cooperative behaviour in groups of molecules. A gel is a long-range cross-linked molecular network that forms spontaneously in a solution when the concentration of solute molecules exceeds a critical value. ${ }^{25}$ Below the critical concentration the solution behaves like a liquid (the sol) and above it, like a viscoelastic solid (the gel). The process of gelation, which can be regulated (most commonly) by varying the solvent concentration or by changing the temperature, is known as a sol-gel phase transition. Among other reasons, it is important for biologists to understand gel formation because the tissues in our body are viscoelastic materials and there are many places in our body that contain gels. The material of the cornea, skeleton joint, lung surface, brain, stomach and intestine are covered by gels. Aspects of the developmental changes seen in connective tissue can be modelled as a sol-gel transition that is driven by an increase in cell density, connectivity or both (Newman et al. 2004).

In general gels are classified by the strength of the crosslinkages. Some gels are cross-linked chemically by covalent bonds, whereas others are cross-linked physically by hydrogen or ionic bonds and by the physical entanglement of polymer

\footnotetext{
${ }^{25}$ Or, as we will see, when the temperature drops below a critical value; there are other routes to gel formation as well.
}

chains (De Gennes 1979; De Rossi et al. 1991). Since they cannot be dissolved again, gels formed by chemical bonding are in a sense irreversible. A chemical gel is formed by a random cross-linking process of monomers via covalent bonding to give rise to a larger and larger molecule and is named a strong gel. On the other hand, a physical gel is formed by ionic bonds. Moderate heating can reversibly dissolve a physically cross-linked gel, which can therefore be called a weak gel.

Many of the natural polymer gels fall into the class of physical gels. Among them, gels formed by red algae have attracted attention because of their varied applications (Pekcan and Kara 2005). We have used a range of techniques for studying the sol-gel transition. ${ }^{26}$ The Flory-Stockmayer and percolation theories are among those that have been developed during the past half century to describe gel formation and model the sol-gel phase transition. ${ }^{27}$ In the language of percolation, one may think of monomers as occupying the vertices of a periodic lattice, and the chemical bonds as corresponding to the edges that, with some probability, join neighbouring vertices at any given moment (Stauffer and Aharony 1994). These groups can be formed by self-organization by obeying either the classical or the percolation picture.

\subsection{Gelation mechanisms}

The conversion of a sol to a gel can be viewed as a phase transition. Consider the example of a material that is a sol at temperatures $\mathrm{T}>\mathrm{Tc}$ and a gel at $\mathrm{T}<\mathrm{Tc}$. The transition is characterized by thermodynamic properties that exhibit a singularity at the transition point. For example, the free energy behaves like $(\mathrm{T}-\mathrm{Tc})^{-\alpha}$ where $\mathrm{Tc}$ is the critical temperature and $\alpha$, the critical exponent, is expected to be a universal constant. The transition from the sol state to the gel state occurs when small clusters link together and create one huge cluster which fills most of the

\footnotetext{
${ }^{26}$ Experimental techniques used for monitoring sol-gel transition must be very sensitive to structural changes and should not disturb the system mechanically. Fluorescence techniques are particularly useful for elucidation of detailed structural aspects of the gels. The technique is based on the interpretation of the change in anisotropy, emission intensity and viewing the lifetimes of injected aromatic molecules to monitor the change in their microenvironment (Birks 1965). These techniques have been successfully used to perform experiments on polymerization and chemical gel formation (Pekcan et al. 1994). Later studies, using pyrene as an extrinsic fluoroprobe, showed that the glass transition both for the linear bulk polymer (Pekcan et al. 1997) and gels (Yilmaz et al. 2002) could be described by a percolation model. In these studies, the fluoroprobe monitors the change occurring in the rigidity of the medium near the glass transition. Photon transmission was used to study the sol-gel and gel-sol transitions of $\kappa$ - and $\mathrm{l}$-carrageenan in pure water and in cationic solution (Kara et al. 2003; Pekcan and Kara 2005).This technique was also used to monitor the gelation of acrylamide (AAm) (Kara and Pekcan 2000) and $\mathrm{N}$ isopropylacrylamide (NIPA) (Kara et al. 2002).

${ }^{27}$ Statistical theories based on tree approximations, which are called mean field or classical theories, originate from Flory (1941) and Stockmayer (1943), and assume equal reactivities of functional groups and the absence of cyclization reactions.
} 
volume. The moment at which the huge cluster just starts to appear indicates the gel point, $p=p_{c}$, where the conversion factor $p$ is the fraction of the bonds which have been formed between the molecules. Thus, the system is called a gel for $p>$ $p_{c}$, a sol for $p<p_{c}$. In the gel state, the number of finite clusters decreases during gelation, whereas the size of the huge cluster grows until all molecules are involved in the infinite network. It is worthwhile noting that the size of the huge cluster, which is called gel fraction, plays the role of the order parameter in the Landau theory of the second-order phase transitions. There are two groups of theories which differ in their treatment of intramolecular loops, namely, space dimensionality and excluded volume effects, in order to describe the sol-gel transition. These are classical theories like those of FloryStockmayer and scaling theories based on lattice percolation. Statistical theories based on tree approximations are called classical theories and originate from Flory (1941) and Stockmayer (1943). They assume equal reactivities of functional groups and the absence of cyclization reactions. Flory and Stockmayer (Flory 1941; Stockmayer 1943) first modelled the sol-gel transition on a special lattice called the Bethe lattice on which closed loops were ignored (figure 1). On the other hand, the gelation mechanism can be modelled employing coupled differential equations, which is called a kinetic model. An alternative to the chemical-kinetic theory is the lattice percolation model (Stauffer and Aharony 1994), where monomers are thought to occupy the sites of a periodic lattice (figure 2).

CLASSICAL THEORY

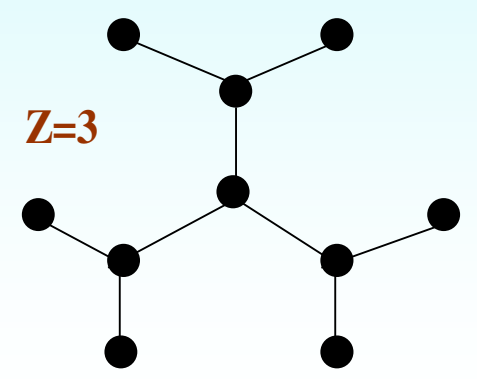

Figure 1. Cayley tree on a Bethe lattice in the classical FloryStockmayer model for the sol-gel transition. Each node is connected to $\mathrm{Z}$ nodes (the coordination number) in this infinite, cycle-free lattice. The filled circles and lines stand for lattice sites and bonds respectively.

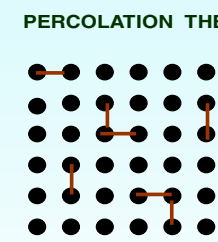

$\mathbf{p}<\mathbf{p}_{\mathbf{c}}$

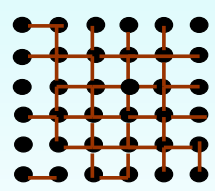

$\mathbf{p}=\mathbf{p}_{\mathrm{c}}$

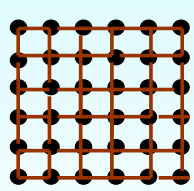

$\mathbf{p}>\mathbf{p}_{\mathrm{c}}$
Figure 2. Lattice percolation model for the sol-gel transition on a square lattice. The filled circles and red lines stand for lattice sites and bonds respectively. $p$ is the probability that a pair of neighbouring lattice sites is connected by a bond and $p_{c}$ is the critical value of $p$ at which percolation takes place (the percolation threshold).

A bond between neighbouring lattice sites is formed randomly with probability $p$. Above a certain bond concentration $p_{c}$ (defined as the percolation threshold), an infinite cluster is formed in the thermodynamic limit. These two models make different predictions in their functional behaviour regarding the values of the critical exponents for the sol-gel transition. Consider, for example the exponents $\gamma$ and $\beta$ for the weight average degree of polymerization, $D P_{w}$ for $p<p_{c}$, and the gel fraction $G$ for $p>p_{c} \cdot{ }^{28}$ Near the gel point these mathematical descriptions are given as

$$
\begin{array}{ll}
D P_{w} \propto\left(p_{c}-p\right)^{-\gamma}, & p \rightarrow p_{c}^{-} \\
G \propto\left(p-p_{c}\right)^{\beta}, & p \rightarrow p_{c}^{+} .
\end{array}
$$

While the Flory-Stockmayer theory gives $\beta=\gamma=1$, independent of dimensionality, percolation studies based on computer simulations give $\gamma$ and $\beta$ around 1.7 and 0.43 in three dimensions (Stockmayer 1943; Stauffer and Aharony 1994). ${ }^{29}$

\subsection{Swelling of gels}

Besides the liquid-like and solid-like aspects that we have just considered, gels can also exhibit a feature of gaseous behaviour: the volume of a gel can rise or fall substantially just like that of a gas that undergoes expansion or compression. The change resembles a phase transition. Polymer networks or gels

\footnotetext{
${ }^{28}$ In percolation language these are the average cluster size and the strength of the infinite network.

${ }^{29}$ Although the molecular structure and the formation of physical gels are rather different from those of chemical gels, the basic properties of the gel state probed by rheological and viscoelastic measurements show close similarities. It is possible to use the theoretical approaches originally derived only for chemical gels in describing the properties of physical gel networks, with some success (Yilmaz et al. 2002; Kara et al. 2003; Pekcan and Kara 2005).
} 
are known to exist generally in two forms, swollen and shrunken (figure 3). Volume phase transitions occur between these forms either continuously or in sudden jumps (Tanaka and Fillmore 1979). The swelling, shrinking and drying kinetics of physical and chemical gels are very important in many technological applications: in the pharmaceutical industry in designing slow-release devices for oral drugs; in the agricultural industry for producing storable foods; and in medical applications in developing artificial organs. Since the gel is formed from a polymeric network, it behaves as an elastic entity. The swelling properties of chemically cross-linked gels can be understood by considering the balance between osmotic pressure and restraining force (Li and Tanaka 1990; Zrinyi and Horkay 1993). The theory of kinetics of swelling for a spherical chemical gel was first developed by Tanaka et al., where, due to the elasticity, the shear modulus plays an important role which keeps the gel in shape by the coupling of any change in different directions. This model predicts that the geometry of the gel is an important factor; and because of the elasticity, swelling is not a pure diffusion process, with the gel presenting collective behaviour during swelling (Li and Tanaka 1990).

Collective cooperative diffusion coefficients were measured by using a fluorescence technique and found to be around $10^{-5} \mathrm{~cm}^{2} / \mathrm{s}$ for PMMA gels swollen in chloroform (Yilmaz and Pekcan 1998). It was observed that during swelling, densely formed gels show smaller time constants than loosely formed gels. This can be understood from the fact that loosely formed gels are more flexible, can take more solvent easily and need longer to reach a fully swollen state.

\subsection{Social analogies to gelation and gel behaviour}

As far as the mathematical description of the sol-gel phenomenon is concerned, both physical and chemical gels obey these models with their proper critical exponents. In the light of the above predictions, one can draw analogies between gels and phenomena of biological, or better social, relevance. Since chemical and physical gels are formed by covalent and ionic bonds, they can be named as strong and weak gels respectively. If one compares monomers to individuals in society, chemical (physical) gels are analogous to strongly (weakly) interacting social groups in a community. Similarly, one can draw an analogy between the swelling of gels and collective behaviour in society. Similar to monomers in a gel, the constituents are simultaneously individuals and a part of society. Neither the individuals in a community nor the monomers in a gel are free; the collective behaviour that they display depends on mutual interactions, on social rules.

It was stated above that variations in gel thicknesses show that loosely formed gels swell much more than densely formed gels. These behaviours of gels suggest the possibility of extending the analogy further. Social communities, too, show differences depending on how loose or tight the relations between individuals are. For instance, some social institutions such as families manifest strong ties between individuals that cannot be loosened easily. The analogy cannot be pushed too far: gel swelling is a reversible phase transition, meaning that if a gel is dried, it can be swollen again. However, social behaviour can be an irreversible process depending on the time and space. If the social structure is destroyed, it is difficult to reverse it.

On the other hand, an epidemic is a contagious disease caused by a biological pathogen and can spread from human to human via a social network. The study of epidemic diseases such as tuberculosis, measles, influenza and sexually transmitted diseases lends itself to 'sol-gel-type' models. The explosive spread of an epidemic has been modelled as bond percolation on the network represented by the social community (Newman 2002). As mentioned, the percolation model can be used to explain the progress of disease spread in spatially organized populations of plants and animals. The Susceptible-InfectedRecovered (SIR) and Susceptible-Exposed-Infected-Recovered (SEIR) models describe the spread of epidemics. We have borrowed ideas from these two well-known models of

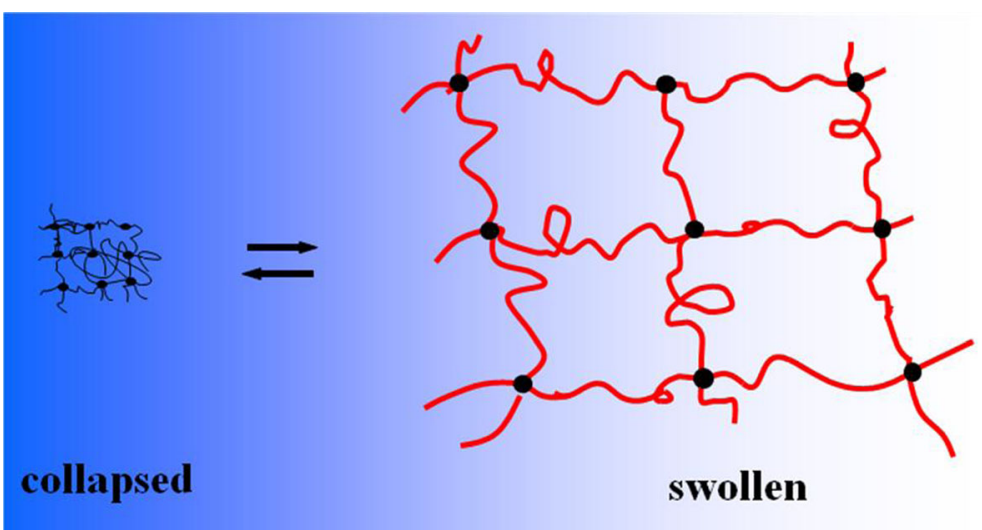

Figure 3. The phenomenon of gel swelling. Shrunken and swollen states of the gel are indicated on the left and right sides of the picture respectively. Filled circles represent cross-linked sites and red lines, polymer chains. 
epidemiology and applied them to phase transitions in physical systems consisting of polyacrylamide (PAAm)-Sodium Alginate (SA) composites, where percolation and classical results were found below and above the certain SA content respectively (Bilge et al. 2012). The differential equations for the SIR and SEIR models were solved over a wide range of parameters and experimental and theoretical results were matched so as to determine the best model and parameter values that fit the observed phase transitions. In fact we modelled the sol-gel transition for a (PAAm)-(SA) composite with different concentrations of SA as SIR and SEIR dynamical systems by solving the corresponding differential equations numerically. It turned out that the SIR model was appropriate for the group of experiments that were labelled as to fit the percolation model. On the other hand, the classical type phase transition model fits the SEIR epidemic model well.

\section{Groups in biology}

In this section we compare and contrast explanations for the behaviour of living and non-living groups. Biological groups consist of living entities that are the products of evolution. Therefore, they can be expected to display features that are peculiar to their evolutionary history, in other words features that are not 'default' consequences of their composition. In this sense they are different from groups made up of non-living entities. At the same time, groups of living organisms cannot function outside the laws of physics, and there may well be features of their behaviour that can be viewed as simple consequences of the laws. Two relevant questions are (a) to what extent some feature of group behaviour demands a specifically biological (=evolutionary) explanation ${ }^{30}$ and (b) at what level is the explanation required. The examples below illustrate this. We begin with a brief introduction to biological evolution. On the way we point out that the most common explanation for evolution invokes genes as the basic units of change but is agnostic with regard to the link between genes and traits and the level(s) at which genetic effects operate. Next we give examples of group behaviour that seem to be accountable (substantially though not entirely) by elementary physical principles and examples of behaviour that are not so easily accountable. The section ends with an overview of evolutionary explanations for group behaviour.

\subsection{Biological organization}

Biology is the study of plants, animals, microbes and viruses - living matter. Living matter possesses characteristics that, taken together, make it unlike the familiar matter of the

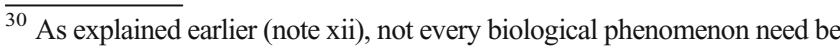
a product of evolution. However, evolutionary (=historical) explanations play a role in biology that they do not in standard physics or chemistry.
}

non-living world. Those characteristics include highly structured configurations that exchange matter, energy and information with the environment and appear to persist stably over long times, and the property of reproduction. It was recognized long ago that living matter lends itself to classification in terms of a nested framework of hierarchical categories; it exhibits different levels of organization. Precisely what the appropriate categories are has been much discussed. But there is general agreement that they include kingdom, phylum, genus and species; and down the level, organisms, tissues and cells. ${ }^{31}$ A phylum comprises a large number of similar species and a species is made up of many similar but not identical organisms - entities that are physically distinct and appear to exhibit autonomous behaviour. An organism itself is made up of one or more cells. The rule of similarity breaks down at the last step: typically the cells of a multicellular organism are very different from one another in form, structure and function (strictly speaking, 'very different' refers to tissues that consist of apparently identical cells). For a biologist, the most striking feature of living forms is that they are highly diverse, and exhibit different kinds of adaptation. 'They have morphologies, physiologies and behaviours that appear to have been carefully and artfully designed to enable each organism to appropriate the world around it for its own life' (Lewontin 1978).

\subsection{Evolution}

Evolution is a fact: all living forms share a common ancestry. Evolution is said to take place when the members of a species differ significantly from their ancestors; and when it does, the long-term stability referred to above breaks down. Darwin and Wallace pointed out that evolution could occur by a process that they called natural selection, which proceeds via heritable variations of minor effect. Small changes accumulating over large times result in large differences between the descendants of very similar organisms. A measure of success (or failure or neutrality) is associated with each modification depending on whether its bearer leaves behind a number of offspring that is larger (or smaller or the same) with respect to the population average; in effect each modification is put to test. Working in this manner, natural selection acts as a locally optimizing process and accounts for the fitness of the organism to its environment, i.e. accounts for adaptation. As a result one can attach a value to the traits possessed by a living entity: the traits appear to be beneficial to their bearers in the sense that most trait modifications would lower the probability that the entity would

\footnotetext{
${ }^{31}$ Organisms, cells, proteins and amino acids belong to the category of 'things that contain other things'. The others have more to do with properties that are common to the members of a category and are useful for distinguishing between categories.
} 
persist in the long run. The origin of the variations is unexplained by natural selection. ${ }^{32}$ So also, whether they are random or directed is a separate issue and has to be decided empirically. Other explanations for evolution include randomness (Kimura 1983; Bonner 2013), abrupt changes of major effect (as happens when individuals belonging to two species of plants hybridize), physical constraints that permit changes only between a small number of discrete alternatives and the moulding by an organism of its environment ('niche construction'). Among these the last outcome is automatically adaptive, whereas the first two need further arguments to account for adaptation. If the environment does not discriminate between different phenotypes, evolution is possible without adaptation. ${ }^{33}$ To get back to evolution by natural selection: it occurs when the members of a species are not all alike, when they differ in their ability to survive and reproduce on account of differences in particular traits, and when they pass on the capacity to develop those traits to offspring. These are testable statements. The modern version of natural selection involves the recognition that heritable differences in traits can be traced to heritable differences in genes, which are often DNA molecules (figure 4$){ }^{34}$

The fact that living forms are linked by common descent allows us to view biology from a historical perspective, something that is not true of standard physics and chemistry. More so, to the extent that natural selection operates, the historical perspective offers an explanatory framework. Both features are captured in a famous saying of Dobzhansky: 'Nothing in biology makes sense except in the light of evolution'. Any feature of the living world can be studied by asking two questions: (a) Through what route could the trait have evolved, i.e. what was it about the trait that, in the past, made its bearers more successful reproducers than their ancestors who lacked the trait? (b) What is responsible for the maintenance of the trait at present? The two questions may have the same answer.

\footnotetext{
$\overline{32}$ Gene mutations constitute one source of variation. As far as we know mutations take place 'at random', meaning that the probability of occurrence of a mutation is independent of its consequence. The physics and chemistry behind the generation of form can be responsible for variations that occur within the same genetic background and the same external environment. Variations can also be induced by the environment. If morphological variations fall within predictable categories, and if variations induced by the environment subsequently find a genetic, and thereby heritable, correlate, patterns of variation may be inherent to the organism's biology and not random; see Müller and Newman (2003).

${ }^{33}$ It may have been important in the evolution of - among other things ammonite shell suture patterns (Raup et al. 1973) and in the morphological evolution of microorganisms (Bonner 2013).

34 The nature of the link between genotype and phenotype falls outside evolutionary theory. In spite of being intensively studied it remains a major problem in biology. The chief reason for this state of affairs is that a large number of factors can influence the phenotype. A partial list would include (a) single ('Mendelian') genes of major effect; (b) many genes; (c) the physical environment; (d) the biological environment; (e) gene-environment correlations; and (f) stochastic effects.
}

But they need not, because evolution in other traits and the relevant physical and biological environments may be different from what it was when the trait first evolved.

\subsection{Groups}

A biological group is a set of entities that constitutes a living organism or forms part of one, is capable of reproducing directly or indirectly and exhibits a degree of coherence that sets it apart from other similar groups. In other words, the group displays a measure of individuality. The hierarchical nature of biological organization means that groups are identifiable at several levels. Examples are chromosomes (a DNA sequence with associated proteins), cells (a genome, possibly contained within a nucleus, other organelles and a cytoplasm), tissues (a set of similar cells, normally in close cohesion), individuals (when multicellular, made up of different tissues) and more familiar groups that go under names such as family, pack or tribe. All of them exhibit coordinated behaviour that indicates a level of autonomy vis-à-vis other similar groups; all of them show long-term persistence in one form or another. Many are capable of reproduction. This is certainly true of DNA sequences, chromosomes, cells and individuals. ${ }^{35}$ The critical point is that it is meaningful to think of a DNA sequence, chromosome, cell or individual reproducing either more or less efficiently than another. ${ }^{36}$ If differential reproductive success is correlated with heritable variation in some trait, and if there are no countervailing forces, the most efficient version of the trait will prevail eventually. The question before us is whether one needs to invoke evolution to explain a particular trait that is expressed at the level of the group (see Houchmandzadeh 2009).

Obviously, the interesting situation is one in which the group displays a trait that is not possessed by its members when considered separately. The first thing to look for is whether the trait resembles a colligative property of a physical system (such as the elevation in the boiling point of a solution as a function of the solute concentration), i.e. is a straightforward consequence of the fact that the group is made up of more than one member.

A purely physical consequence of group formation may be biologically beneficial to each member of the group. Many DNA sequences that are strung together and compacted may be less accessible to degradation by

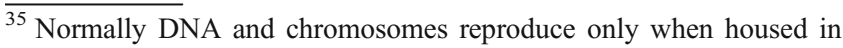
cells. On the other hand cells and multicellular organisms do so autonomously. But that too is possible only in an appropriate environment (which however does not reproduce).

${ }^{36}$ In the case of cells and multicellular organisms the point is obvious. The phenomena of meiotic drive and segregation distortion provide examples of a DNA sequence or chromosome being more likely to be transmitted through meiosis than another (usually the homologue; see Taylor and Ingvarsson 2003).
} 


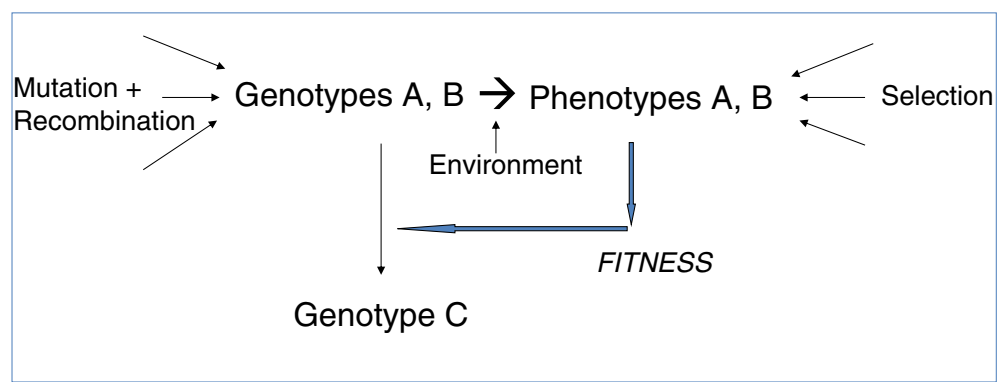

Figure 4. The neo-Darwinian picture of natural selection in the case of organisms that reproduce sexually. The genotype and the environment, taken both separately (in principle) and in concert, specify the phenotype. Traditionally, evolutionary change is believed to be based on that part of inter-individual phenotypic variation that is attributable to differences in genotype. The evolutionary origin of a trait and its maintenance require to be considered independently (see Linde-Medina and Newman 2014 for alternative views of evolution).

nucleases than the same sequences on their own. A bird that flies in a flock could gain more protection against a raptor than a bird that flies on its own. The compactness of a group may be the result of each member trying to be maximize its distance from the periphery - again, to avoid predation (Hamilton 1971). By huddling together, rat pups decrease their overall surface:volume ratio and reduce heat loss; thereby they reduce the energy required for maintaining body temperature (Alberts 1978). An increase in number per se can lead to beneficial consequences. Some cellular slime mould amoebae form fruiting bodies consisting of a single spore held up by a filamentous stalk. If each of $n$ amoebae that do so happen to come together, the load that requires to be supported goes up in proportion to $n$ but the capacity of the stalk to support the load goes up as $n^{2}$, a factor that could have potentiated the aggregation of unicellular amoebae. In a beehive, as the number of foragers $(n)$ goes up, the forage gathered increases in proportion. But relative to the mean, the extent to which it varies increases only as the square root of $n$, so that the relative variation decreases as $(1 / \sqrt{ } n)$. It has been suggested that since this helps in improving the economy of the hive - by making the supply of forage more predictable - it could have potentiated the evolution of sociality in bees (Stevens et al. 2007).

There are two points to be noted in these examples. (a) First, the groups were homogeneous - the trait of interest was the same in each member, and the gains of group living were distributed equally. This is unlikely to be true in general. In contrast to physical groups, that at a certain fundamental level can often be thought of as being made up of identical subunits (discussed in the first section of this article), inter-individual variation is a central feature of living systems. Even if the individuals in a group have the same genetic makeup, their phenotypes can differ - even in the same environment and, one might add, at the same stage of the life cycle. The plasticity of the phenotype confers a degree of flexibility to biological groups that physical groups lack (Nanjundiah 2003) and increases the likelihood of emergent phenomena (Kaneko 2006). When it exists, genetic variation is an additional element that contributes to phenotypic variation. Because of phenotypic variation, the costs and benefits of group living need not be shared equally. (Indeed, in extreme cases, for example, in cellular slime moulds and ants, group life may make it impossible for some individuals to reproduce, meaning that their fitness is zero.) (b) Second, in arguing that group life can provide a purely physical advantage we do not exclude a biological basis behind the advantage. In other words, group formation may have been favoured by natural selection in the past; having evolved, group structure may be maintained by natural selection in the present. Physical and biological inputs can reinforce each other too. Natural selection can give rise to groups of individuals of different phenotypes, whose longterm coexistence may depend on the stability of the underlying network of interactions between them and on trade-offs between fitness-related traits. This seems to be the case in some cellular slime moulds (Sathe et al. 2013).

In the context of groups, there is an unconventional aspect to the genotype-phenotype link which bears on how natural selection can act. The phenotype of an individual is a reflection of its own genotype. But it can be influenced also by the phenotype, and therefore the genotype, of another individual belonging to the same group that interacts with it (a case of an 'extended phenotype'; Dawkins 1982). Since the situation is symmetric, self-reinforcing (positive) feedback can take place. This lays the ground for the group as a whole to exhibit abrupt changes in behaviour akin to a phase transition. Also, because the second individual is a part of the environment of the first, long-term heritable changes in genotype amount to gene-environment co-evolution. The concepts of 'non-autonomy' and 'social selection' are useful for describing the operation of natural selection under these circumstances (discussed in Nanjundiah and Sathe 2011).

Assuming natural selection to be a significant factor in the evolution of groups, at what level is its role significant? Is it 
more likely to favour some genes over other genes, some individuals over other individuals or some groups over other groups? Lewontin (1970) made use of the example of the tlocus in the mouse to show that all three levels are plausible (Nizak et al., in preparation). The example of the t-locus shows that selection can act at the group level, but it does not say anything about group structure or group behaviour. Group structure must lead to at least some consequence that goes beyond what might result as a passive effect of an increase in size or number. For that, we need to invoke interactions between the members of a group. A characteristic feature of biological groups is that they involve interactions between members along with the emergence of nonequivalence or division of labour between them. Division of labour is often accompanied by differential reproduction, which leads to the question of how a stable group structure can be maintained over generations. The t-locus example shows that stable polymorphisms can persist even when some individuals reproduce less effectively than others. But the explanation has to be extended to the maintenance of the group as such.

\section{Summing up}

The phenomenon of individual units interacting with each other and exhibiting novel behaviours is common in physics, chemistry and biology. In physical systems, matter above the level of elementary particles exhibits group-level properties at a series of different levels that extend to the properties of bulk matter familiar to us from everyday experience. A proper understanding of the latter requires the application of relativistic quantum field theory. It is not possible to derive the physics of daily experience by taking the so-called classical limit in which Planck's constant vanishes. The gel transition is a simple example of collective behaviour in groups of molecules that can be triggered abruptly even though the properties of the system (e.g. concentration or temperature) change smoothly. There are interesting analogies between the behaviour of gels and the behaviour of social groups. In an inversion of the usual approach, a biological (epidemiological) model can clarify the dynamics of a sol-gel transition. Living groups permit an additional mode of explanation that is based on their evolutionary history. Where natural selection is the appropriate explanation, one can ask whether variations in aspects of group behaviour can be correlated with genetic variations between individuals and, if so, whether they influence longevity or reproduction or both. The long-term differential survival of genes can be influenced by traits at the level of the chromosome, the individual or the group. At the same time, self-organization is a powerful principle in physics, chemistry and biology, and it remains an open question whether instances of group behaviour can be explained by self-organization too.

\section{Acknowledgements}

We are grateful to Stuart Newman for several helpful comments on a pre-submission draft.

\section{References}

Alberts JR 1978 Group regulatory behavior in developing rats. $J$. Comp. Physiol. Psychol. 92 231-246

Anderson P 1972 More is different. Science 177 393-396

Arpağ G and Erzan A 2014 An ensemble approach to the evolution of complex systems. J. Biosci. 39 259-280

ATLAS Collaboration 2012 Combined search for the Standard Model Higgs boson using up to $4.9 \mathrm{fb}^{\wedge}\{-1\}$ of pp collision data at $\backslash$ sqrt $\{7\} \mathrm{TeV}$ with the ATLAS detector at the LHC. Phys. Lett. B doi:10.1016/j.physletb.2012.02.044

Berry MV 2002 Singular limits. Phys. Today May 10-11

Bilge AH, Pekcan Ö and Gürol MV 2012 Application of epidemic models to phase transitions. Phase Trans. 851009 1017

Birks JB 1965 Photophysics of aromatic molecules (London: Wiley-Interscience)

Bonner JT 2013 Randomness in evolution (Princeton: Princeton University Press)

CMS Collaboration 2012 Observation of a new boson at a mass of $125 \mathrm{GeV}$ with the CMS experiment at the LHC. Phys. Lett. doi:10.1016/j.physletb.2012.08.021

Dawkins R 1982 The extended phenotype (Oxford University Press)

De Gennes PG 1979 Scaling concepts in polymer physics (Ithaca and London: Cornell University Press)

De Rossi D, Kajiwara K, Osada Y and Yamauchi A 1991 Polymer gels (New York: Plenum Press)

Ehrenfest P 1933 Phase changes classified according to the singularities of the thermodynamic potential. Proc. R. Acad. Sci. Amsterdam 36153

Flory PJ 1941 Molecular size distribution in three dimensional polymers. I. Gelation. J. Am. Chem. Soc 63 3083-3090

Frege G 1884 Die grundlagen der arithmetik (Jena)

Hamilton WD 1971 Geometry for the selfish herd. J. Theor. Biol. 31 295-311

Houchmandzadeh B 2009 Theory of neutral clustering for growing populations. Phys. Rev. E 80051920

Kara S, Tamerler C, Bermek H and Pekcan Ö 2003 Cation effects on sol-gel and gel-sol phase transitions of k-carrageenan-water system. Int. J. Biol. Macromol. 31 177-185

Kara S, Okay O and Pekcan Ö 2002 Real-time temperature and photon transmission measurements for monitoring phase separation during the formation of poly( $\mathrm{N}$-isopropylacrylamide) gels. J. Appl. Polym. Sci. 86 3589-3595 
Kara S and Pekcan Ö 2000 Lattice heterogeneities at various crosslinker contents; a gel swelling study. Polymer 41 8735-8739

Kaneko K 2006 Life: An introduction to complex systems biology (Springer)

Kimura M 1983 The neutral theory of molecular evolution (Cambridge: Cambridge University Press)

Kulkarni P, Shiraishi T and Kulkarni RV 2013 Cancer: Tilting at windmills? Mol. Cancer 12 108-111 (http://www.molecularcancer.com/content/12/1/108)

Lewontin RC 1970 The units of selection. Ann. Rev. Ecol. Syst. 1 $1-18$

Lewontin RC 1978 Adaptation. Sci. Am. 239 212-230

Li Y and Tanaka T 1990 Kinetics of swelling and shrinking of gels. J. Chem. Phys. 92 1365-1371

Lieb E and Thirring W 1975 Bound for the kinetic energy of fermions which proves the stability of matter. Phys. Rev. Lett.35 687-689

Lieb E 1990 From atoms to stars. Gibbs lecture. Bull. Am. Math. Soc. 221

Linde-Medina M and Newman SA 2014 Limb, tooth, beak: Three modes of development and evolutionary innovation of form. $J$. Biosci. 39 211-223

Müller GB and Newman SA 2003 Origination of organismal form. Beyond the gene in developmental and evolutionary biology (MIT Press)

Nanjundiah V 2003 Phenotypic plasticity and evolution by genetic assimilation; in Origination of organismal form. Beyond the gene in developmental and evolutionary biology (eds) GB Müller and SA Newman (MIT Press) pp 245-263

Nanjundiah V 2005 Mathematics and biology. Curr. Sci. 88 388-393

Nanjundiah V and Sathe S 2011 Social selection and the evolution of cooperative groups: The example of the cellular slime moulds. Integr. Biol. doi:10.1039/c0ib00115e

Newman SA and Comper WD 1990 'Generic' physical mechanisms of pattern formation. Development 110 1-18

Newman MEJ 2002 Spread of epidemics on networks. Phys.Rev. E 66016128

Newman SA, Forgacs G, Hinner B, Maier CW and Sackmann E 2004 Phase transformations in a model mesenchymal tissue. Phys. Biol. 1 100-109

Pauli W 1940 The connection between spin and statistics. Phys. Rev. 58 716-722

Pekcan O and Kara S 2005 Cation effect on thermal transition of iota-carrageenan: a photon transmission study. J. Bio. Sci. Polym. Ed. 16 317-333

Pekcan Ö, Yilmaz Y and Okay O 1994 Fluorescence technique for studying the sol-gel transition in the free-radical crosslinking copolymerization of methyl methacrylate and ethylene glycol dimethacrylate. Chem. Phys. Lett. 229 537-540

Pekcan Ö, Yilmaz Y and Okay O 1997 Real time monitoring of polymerization rate of methyl methacrylate using fluorescence probe. Polymer 38 1693-1698

Pines D and Alpar MA 1985 Superfluidity in neutron stars. Nature 31627

Raup DM, Gould SJ, Schopf TJM and Simberloff DS 1973 Stochastic models of phylogeny and the evolution of diversity. $J$. Geol. 81 525-542

Russell B 1961 History of Western philosophy (Unwin University Books) p.784.

Saçlığlu C 2003 The quantum mechanical foundations of philosophy. ARI Bull. Istanbul Tech. Univ. 531

Sathe S, Khetan N and Nanjundiah V 2013 Interspecies and intraspecies interactions in social amoebae. J. Evol. Biol. doi:10.1111/jeb.12298

Schrödinger E 1944 What is life? (Cambridge University Press)

Stanley HE 1971 Introduction to phase transitions and critical phenomenon (New York and Oxford: Oxford University Press)

Stauffer D and Aharony A 1994 Introduction to percolation theory (London: Taylor and Francis)

Stevens MI, Hogendoorn K and Schwarz MP 2007 Evolution of sociality by natural selection on variances in reproductive fitness: evidence from a social bee. BMC Evol. Biol. 7 153-

Stockmayer W 1943 Theory of molecular size distribution and gel formation in branched- chain polymers. J. Chem. Phys. 11 45-54

Tanaka T and Fillmore DJ 1979 Kinetics of swelling of gels. $J$. Chem. Phys. 70 1214-1218

Taylor DR and Ingvarsson PK 2003 Common features of segregation distortion in plants and animals. Genetica 117 $27-35$

Wigner EP 1939 On unitary representations of the inhomogeneous Lorentz group. Ann. Math. 40 149-204

Wigner EP 1960 The unreasonable effectiveness of mathematics in the natural sciences. Commun. Pure Appl. Math. 13 1_14

Yilmaz Y, Erzan A and Pekcan O 2002 Slow regions percolate near glass transition. Eur. Phys. J. E 9 135-141

Yilmaz Y and Pekcan O 1998 In situ fluorescence experiments to study swelling and slow release kinetics of disc-shaped poly(methyl methacrylate) gels made at various crosslinker densities. Polymer 39 5351-5357

Zrinyi M and Horkay F 1993 Studies on the swelling and shrinking kinetics of chemically crosslinked disk-shaped Poly(vinyl acetate) gels. Macromolecules 26 3097-3102 